\title{
Charge transport in two different conductive polymer and semiconducting quantum dot nanocomposite systems
}

\author{
Sushmita Biswas, ${ }^{1}$ Yang Li, ${ }^{1}$ Michael A. Stroscio, ${ }^{1-3}$ and Mitra Dutta ${ }^{1,3, a)}$ \\ ${ }^{1}$ Department of Electrical and Computer Engineering, University of Illinois at Chicago, Chicago, \\ Illinois 60607, USA \\ ${ }^{2}$ Department of Bioengineering, University of Illinois at Chicago, Chicago, Illinois 60607, USA \\ ${ }^{3}$ Department of Physics, University of Illinois at Chicago, Chicago, Illinois 60607, USA
}

(Received 17 July 2010; accepted 10 December 2011; published online 24 February 2012)

\begin{abstract}
Charge transport mechanisms have been investigated in two different nanocomposite structures made of conductive polymers embedded with II-VI semiconducting quantum dots. Photoluminescence data indicated charge transport in the two systems. Higher photocurrents observed in the poly (3-hexylthiophene-2,5-diyl) polymer-based heterostructure in comparison with the poly(2-methoxy-5 -(2'-ethyl-hexyloxy)-1,4-phenylenevinylene) polymer-based heterostructure have been analyzed with hole and electron conduction. For larger concentrations of quantum dots, both electron transport through nanocrystals and hole transport through polymer become relevant. Based on the electron tunneling mechanism, current voltage characteristics are modeled for a double barrier quantum well device formed by semiconducting quantum dots and polymer molecules. (c) 2012 American Institute of Physics. [doi:10.1063/1.3682106]
\end{abstract}

\section{INTRODUCTION}

Conductive polymers have emerged as a novel class of electronic materials. The advantages they offer in terms of tunable conductivity, low cost, and flexibility have stimulated a lot of interest in the fields of electronic and optoelectronic devices. ${ }^{1,2}$ With the advent of better design and fabrication technology, a wide selection of conductive polymers is available for applications in optoelectronic devices. High performance light-emitting diodes (LEDs), solar cells, lasers, and transistors have been developed with conductive polymers. ${ }^{1-4}$

Conductive polymer-based nanocomposite systems incorporating semiconducting quantum dots (QDs) have been investigated by a growing number of researchers. ${ }^{1,3,4}$ Of particular interest are the nanocomposites based on poly(2-methoxy-5(2'-ethyl-hexyloxy)-1,4-phenylenevinylene) (MEH-PPV) and poly(3-hexylthiophene-2,5-diyl) (P3HT). The enhanced charge separation at the polymer-QD interfaces make these heterostructures promising for high efficiency photovoltaic and photodetector applications. The efficient collection of separated charge carriers by the electrodes without undergoing recombination is a challenge which requires that the electrons and holes have separate continuous pathways to the electrodes without the need of passing through the other material. The charge collection also needs to be fast, so that electrons and holes have fewer chances to undergo non-radiative recombination at the interfaces. To improve the external quantum efficiencies (number of electrons collected in the external circuit per unit number of incident photons) of the devices based on these conductive polymers, there is a need for a better understanding of the charge transport processes in these hybrid structures.

\footnotetext{
${ }^{\text {a) }}$ Author to whom correspondence should be addressed. Electronic mail: dutta@uic.edu.
}

In this paper, we made an effort to elucidate the charge transport processes in these hybrid structures by making a comparative study in two different nanocomposite systems made of conducting polymers MEH-PPV and P3HT embedded with cadmium selenide (CdSe) QDs. We chose MEHPPV and P3HT as hole transport layers due to the high hole mobilities in these two polymers. MEH-PPV and P3HT form type II heterojunctions with CdSe QDs, favorable for charge transport. We investigated the different components of charge transport based on the experimental and modeling studies of the two different hybrid systems.

\section{A. Fabrication and experiments}

A composite structure is fabricated with MEH-PPV and CdSe QDs by mixing the polymer and the QD solutions and spin casting a layer on indium tin oxide (ITO)-coated glass substrate. A schematic diagram of the fabricated device is displayed in Fig. 1(a). The weight to volume (w/v) ratio of the MEH-PPV solution in chloroform is $10 \mathrm{mg} / \mathrm{ml}$. The CdSe dot solution is made in chloroform with a w/v ratio of $1 \mathrm{mg} / \mathrm{ml} .^{5}$ The concentration of CdSe dots in chloroform is $10^{17} \mathrm{~cm}^{-3}$. The weight percentage of QDs mixed in polymer is $\sim 70 \%$. The mixture was filtered using a sub-micron filter to remove large aggregates. The filtrate was sonicated to ensure that the polymer and the QDs intermix. Interpenetrating networks with nanophase domains have been obtained with this technique. ${ }^{6}$ The top contact is made of gold $(\mathrm{Au})$ pixels (thickness $30 \mathrm{~nm}$ ) with approximately $1 \mathrm{~mm}$ diameter and deposited by a shadow masking technique.

Another hybrid structure is fabricated with P3HT and CdSe QDs by mixing P3HT solution and CdSe QD solution with 70\% weight of QDs in polymer (same technique followed as for MEH-PPV composite) and spin-casting a layer of the mixture on ITO-coated glass substrate. A schematic diagram of the fabricated device is displayed in Fig. 1(b). 

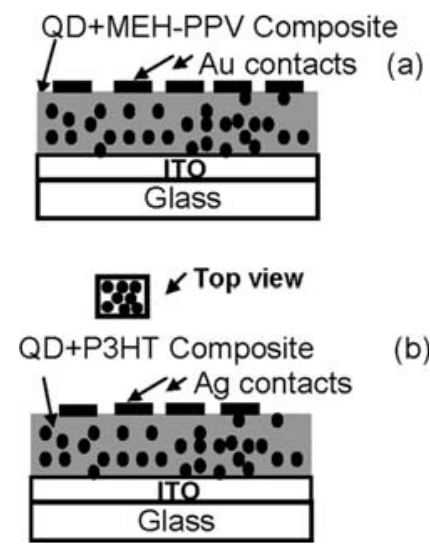

(b)

FIG. 1. Schematic diagram of (a) MEH-PPV/CdSe nanocomposite sample and (b) P3HT/CdSe nanocomposite sample.

The P3HT solution is made in a chloroform solvent with a $\mathrm{w} / \mathrm{v}$ ratio of $10 \mathrm{mg} / \mathrm{ml}$. The CdSe dot solution is made in chloroform with a w/v ratio of $1 \mathrm{mg} / \mathrm{ml}$. The top contact is made with silver (Ag) pixels with diameter approximately 1 $\mathrm{mm}$.

Optical characterization of the samples is performed by photoluminescence (PL) measurements using an Acton SpectraPro 2500 spectrometer, and the samples are excited by either an $\mathrm{Ar}^{+}$ion or a He-Cd laser. Current voltage (I-V) characteristics are measured on the two samples at room temperature using a probe station and an HP4156B semiconductor parameter analyzer.

\section{RESULTS AND DISCUSSION}

Optical characterization is performed on the two samples (MEH-PPV/CdSe and P3HT/CdSe) to see effects of charge transport on the PL properties of the polymers and QDs. In Fig. 2(a) is shown the PL data of the MEH-PPV/CdSe sample excited with a $442 \mathrm{~nm}$ laser excitation. The emission due to the polymer is substantially quenched in this sample due to the addition of CdSe QDs. The PL data of the pure MEH-PPV polymer is shown in Fig. 2(a), and that of CdSe QDs is shown in Fig. 2(b). The PL data from the P3HT/CdSe sample is shown in Fig. 3(a). The PL from P3HT polymer is also shown in Fig. 3(a). The emission from pure CdSe QDs is shown in Fig. 3(b). The polymer PL is quenched due to the presence of the CdSe QDs, which is an indication of charge separation at the QD/polymer interfaces. ${ }^{7}$

Morphological studies made on such composite systems fabricated with a similar approach have shown that the polymer and the QDs form nanophase domains. ${ }^{6}$ The formation of these nanophase domains is important for an efficient charge separation process. The photogenerated excitons need to undergo charge separation at the interfaces before undergoing recombination. The exciton diffusion lengths in conjugated polymers are typically in the range of $5-15 \mathrm{~nm} .^{7}$ Thus, in order for excitons to meet the interface before undergoing recombination, nanophase morphology is important.

In charge transport processes, the luminescence from the polymer is quenched as photogenerated excitons are dissociated at the interface between the QDs and the polymer. Also,
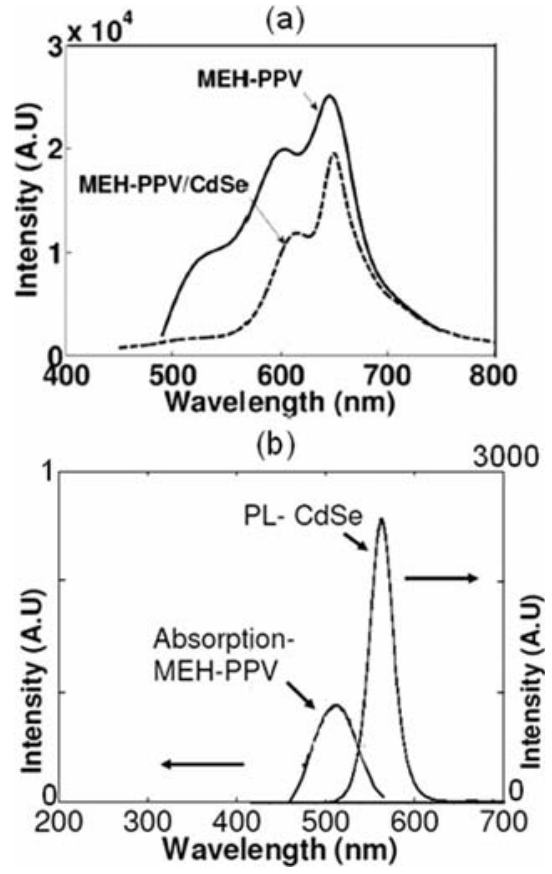

FIG. 2. (a) PL from the MEH-PPV/CdSe sample at room temperature (dashed line). PL from MEH-PPV film (dark line). (b) PL from CdSe QDs film and absorption from MEH-PPV film. Excitation laser wavelength is at $442 \mathrm{~nm}$.

there is an increase in the natural radiative lifetime in the composite as compared to the pure polymer. ${ }^{8}$ This implies that the excitons in the polymer which contributes to the remaining $\mathrm{PL}$ in the composite migrate along the polymer chain a longer distance before recombination. The PL spectra of the composites appear similar to that of the polymer, and

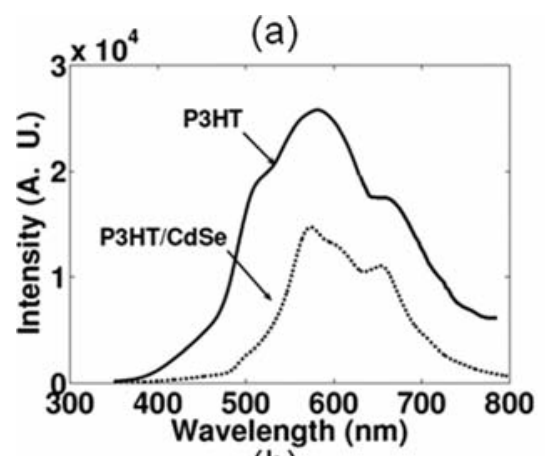

(b)

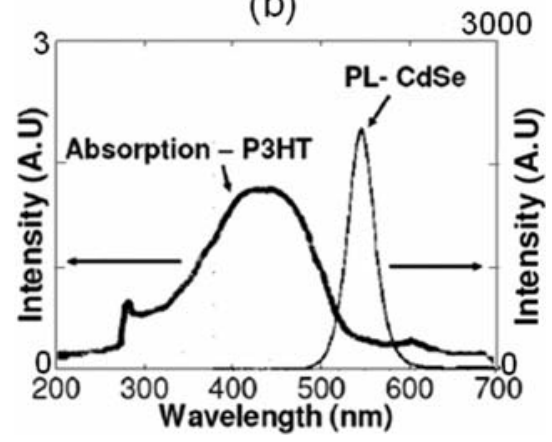

FIG. 3. (a) PL from P3HT film (dark line). P3HT dissolved in chloroform with $10(\mathrm{mg} / \mathrm{ml})$. PL from the P3HT/CdSe sample at room temperature (dashed line). (b) PL from CdSe QDs and absorption from P3HT. 
this indicates that the remaining PL is predominantly from the excitons recombining radiatively in the polymer.

The charge transfer from polymer to QDs can be explored with the help of electron affinities (EA) of the polymer and the QDs. ${ }^{7}$ Charge transfer from polymer to QDs is energetically favorable if the following condition is satisfied:

$$
\mathrm{EA}_{Q D}-\mathrm{EA}_{P O L}>\mathrm{U}_{P O L}-\mathrm{V}_{C T},
$$

where $E A_{Q D}$ and $E A_{P O L}$ are the electron affinities for the QD and the polymer, $U_{P O L}$ is the Coulombic binding energy of the photogenerated exciton in the polymer, and $V_{C T}$ is the Coulombic attraction between the separated electron and hole. In general, $U_{P O L}$ is greater than $V_{C T}$, due to the increased separation between the charges after exciton dissociation. The EA values for CdSe QDs, MEH-PPV, and P3HT are $3.7 \mathrm{eV}$, $2.8 \mathrm{eV}$, and $3.2 \mathrm{eV},{ }^{9-12}$ respectively. The difference in the electron affinities are $0.9 \mathrm{eV}$ in the $\mathrm{MEH}-\mathrm{PPV} / \mathrm{CdSe}$ and 0.5 $\mathrm{eV}$ in $\mathrm{P} 3 \mathrm{HT} / \mathrm{CdSe}$ nanocomposites. The reported excitonbinding energies in MEH-PPV and P3HT are $0.2 \mathrm{eV}$ and 0.5 $\mathrm{eV}$, respectively. ${ }^{13,14}$ It shows that electron transport is possible from the polymer to the QDs in both the samples. This is consistent with the observed quenching of the PL corresponding to the polymer peak in the nanocomposite samples.

It is observed from Fig. 2(b) that there is an overlap of the emission spectrum of the CdSe QDs and the absorption spectrum of MEH-PPV and P3HT. This gives an indication of a possible energy transfer between QDs and the polymer. ${ }^{15}$ However, in energy transfer processes, the luminescence from the QDs should quench as the energy is transferred from the QDs to the polymer molecules with a corresponding increase in the polymer PL, ${ }^{16}$ which is not observed in our results. Thus, although a probability of energy transfer exists in these two systems, it is not clearly observed. This could be due to the charge separation at the interfaces of QDs and polymer. Thus, a competition develops between the charge transfer and the energy transfer processes in the QDs and the polymer molecules in the two nanocomposite systems. ${ }^{17}$

I-V measurements are also performed to study the charge transport properties in the two structures. In Fig. 4(a), the I-V curves are plotted for the MEH-PPV/CdSe sample for the $325 \mathrm{~nm}$ and $442 \mathrm{~nm}$ laser excitations and for the dark condition. The I-V curves are repeatedly consistent within a small error equal to one standard deviation between successive measurements, as shown in Fig. 4(a). Forward bias currents under higher magnification are shown (bottom right). It reveals that the photocurrents are greater than the dark current, but not as high as reported by other groups. ${ }^{7}$ A possible reason for this may be the role of interface states in trapping the photogenerated charge carriers, which eventually undergo nonradiative recombination if they are not collected by the external applied field. Interface dipoles may also pose additional barriers to charge transport. The nature of interfaces of the QDs depends on the surrounding organic ligands to a large extent. The details on the ligands used in the synthesis of these QDs are provided elsewhere. ${ }^{5}$ The photodiode nature of the device is revealed by the asymmetry in the I-V curves, arising due to the difference in the work functions of the contact metals (ITO and Au). An internal electric field (a)

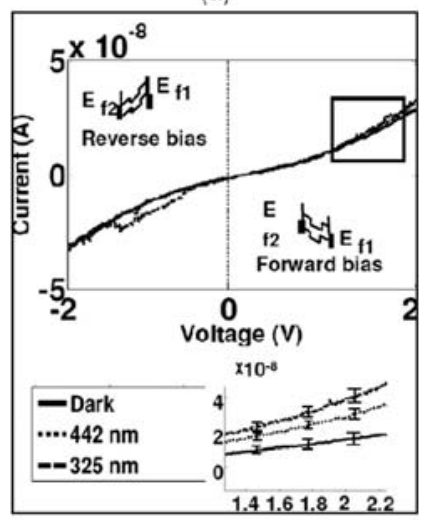

(b)

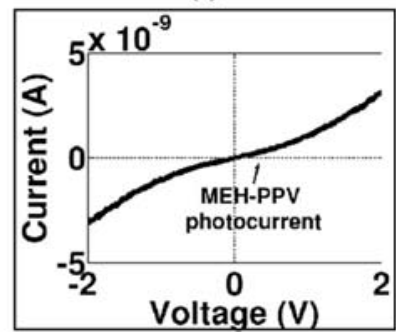

FIG. 4. I-V characteristics for (a) MEH-PPV/CdSe nanocomposite sample at room temperature. Dark line represents dark current and dotted line represents photocurrent with a $442 \mathrm{~nm}$ laser and dashed line represents photocurrent with a $325 \mathrm{~nm}$ laser. The forward and reverse bias band alignments are shown in the insets. Region inside the rectangular box is magnified and shown at the bottom right. Error bars represent one standard deviation between measurements. (b) MEH-PPV film.

generated due to this difference helps in the extraction of the separated charge carriers toward the opposite electrical contacts and is necessary for good photovoltaic efficiency. However, in a photodetector, an external field is applied to collect the separated charge carriers.

The current voltage characteristics of the P3HT sample are shown in Fig. 5(a). The photocurrent generated in this sample is higher compared to the MEH-PPV sample by approximately six orders of magnitude. The forward-biased currents under higher magnification are also shown in Fig. 5(a) (bottom). It reveals that the photocurrent for the $325 \mathrm{~nm}$ laser excitation is higher than that for the $442 \mathrm{~nm}$ laser excitation as well as the dark current. The $325 \mathrm{~nm}$ laser excites the electrons in the QDs as well as in the polymer molecules, causing an increase in the output current. It is also observed that the photocurrent at $442 \mathrm{~nm}$ laser excitation is smaller than the dark current. This is because P3HT absorption peaks at around this wavelength, and it forms Frenkel excitons, which do not dissociate until around $4 \mathrm{~V}$, due to their high binding energies.

The observed six order increase in the photocurrents is first investigated with hole mobilities of the two polymers. It is known that MEH-PPV and P3HT are p-type polymers. ${ }^{1,18}$ The hole mobilities in the P3HT and MEH-PPV are $\sim 10^{-3}$ and $\sim 10^{-6} \mathrm{~cm}^{2} / \mathrm{V}$.s, respectively. ${ }^{19,20}$ There is a difference of approximately three orders in the hole mobilities. Moreover, the photocurrent in MEH-PPV is in the order of $\sim$ nanoampere, ${ }^{21}$ as observed from Fig. 4(b). Upon addition of the CdSe QDs, there is an increase of an order 
(a)

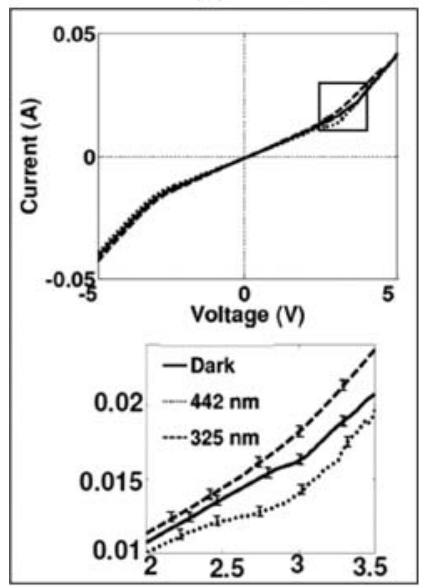

(b)

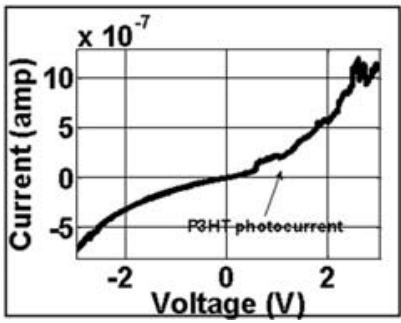

FIG. 5. I-V characteristics for (a) P3HT/CdSe nanocomposite sample at room temperature. Dashed line represents photocurrent for a $325 \mathrm{~nm}$ laser excitation, dotted line for $442 \mathrm{~nm}$ laser excitation, and dark line represents dark current. Region inside the rectangular box is magnified and shown at the bottom. Error bars represent one standard deviation between measurements. (b) P3HT film.

in the photocurrents, as observed in our nanocomposite of MEH-PPV and CdSe. In P3HT, the photocurrent is in the order of microampere, ${ }^{22}$ as observed from Fig. 5(b). However, in the nanocomposite of $\mathrm{P} 3 \mathrm{HT}$ with $\mathrm{CdSe}$, there is a four orders of magnitude increase in the photocurrent. The ratio of the photocurrents in the composite and the polymer is much higher in the $\mathrm{P} 3 \mathrm{HT} / \mathrm{CdSe}$ case compared to the MEH-PPV/CdSe case. Thus, there is an unequal scaling of currents upon addition of the same weight percentage of $\mathrm{CdSe}$ in the two polymers. This indicates that hole conduction alone cannot explain the observed six order increase in the currents in the P3HT composite. There could be other mechanisms of current conduction in addition to the hole conduction. Hence, we investigated the electron conduction through the QDs. For a large fraction of QDs in the polymer, they can form transport channels for electrons. Since the concentration of QDs is high in the polymers $(\sim 70 \%$ weight), it is possible that the electron component of the current, by tunneling through the nanocrystals (NCs), is a significant component of the overall current.

Proposed energy band alignments of the QDs and the polymer molecules in the two nanocomposite samples at equilibrium are shown in Figs. 6(b) and 6(d), where the dopant levels are unknown. For this energy band alignment, the HOMO and LUMO levels chosen for MEH-PPV are at -5.3 $\mathrm{eV}$ and $-2.8 \mathrm{eV}$, and, for P3HT, the levels are at $-5.1 \mathrm{eV}$ and $-3.2 \mathrm{eV}$. All the energies are with respect to vacuum level, which is not shown in these figures. Figures 6(a) and 6(c) depict the simplest possible non-rectifying junctions formed at the interfaces of ITO/P3HT, P3HT/Ag and ITO/MEH$\mathrm{PPV}, \mathrm{MEH}-\mathrm{PPV} / \mathrm{Au}$ junctions. $\mathrm{Au}$ and $\mathrm{Ag}$ form nonblocking contact with MEH-PPV and P3HT, respectively. $^{23,25}$ The bands are aligned by raising the Fermi level of ITO and lowering it down for Au (opposite directions for the ITO and Ag). Typical values are chosen for the conduction band minimum and the valence band maximum for CdSe (Ec -3.7 eV, Ev -5.8 eV) QDs. ${ }^{12}$ During forward bias, the electrons and holes do not encounter a barrier due to the tilted band and are collected by the Au (Ag in case of P3HT/ $\mathrm{CdSe}$ ) electrode and the ITO electrode, respectively, in the MEH-PPV/CdSe sample, as shown in Fig. 4(a). Reverse bias causes photogenerated electrons and holes to move downhill along the tilted band. Electrons are collected by the ITO electrode, and holes are collected by the $\mathrm{Au}$ ( $\mathrm{Ag}$ in the case of P3HT/CdSe) electrode. The difference in the Fermi energies of the contacts (ITO $-4.8 \mathrm{eV}, \mathrm{Au}-5.1 \mathrm{eV}$ ) results in an equilibrium upward tilt in the bands in the MEH-PPV/CdSe sample. In the P3HT/CdSe sample, the difference between the Fermi energies of the contacts (ITO $-4.8 \mathrm{eV}$ and $\mathrm{Ag}-4.7$ $\mathrm{eV})$ is small $(0.1 \mathrm{eV})$ and is not shown in the band diagram in Fig. 6(b). The effect of this tilt is small and is observed in the small asymmetry in the I-V curves. It is seen that the energy offsets for the conduction bands of the QDs and the polymers are smaller for the P3HT/CdSe sample. The confinement is less pronounced in the quantum well of this structure, as compared to that of MEH-PPV/CdSe. This leads to higher probabilities of electron tunneling through this structure than MEH-PPV/CdSe. Additional band offsets might be present, due to the presence of interface dipoles as well as interface traps serving as charged centers. In this proposal for band alignment, the band offsets, which may be present due to interface properties, are ignored.

A type II heterostructure is formed by the polymer and the QD materials in both the samples, which favors the (a)

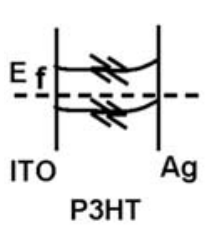

(c) (b)

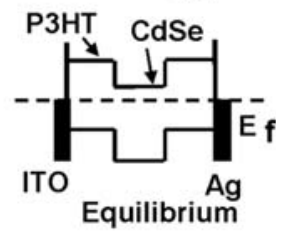

(d)

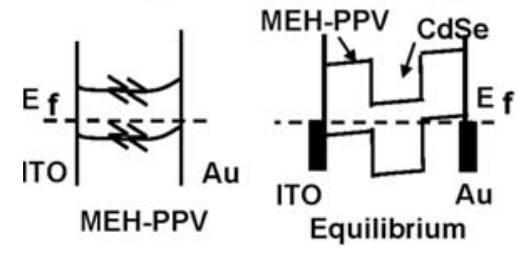

FIG. 6. Energy band diagrams for the metal and polymer junctions. (a) ITO/ $\mathrm{P} 3 \mathrm{HT} / \mathrm{CdSe} / \mathrm{Ag}$-ohmic junctions at ITO/P3HT and P3HT/Ag interfaces. (b) Equilibrium energy band diagram for P3HT/CdSe sample. (c) ITO/MEH$\mathrm{PPV} / \mathrm{Au}-\mathrm{ohmic}$ junctions at ITO/MEH-PPV and MEH-PPV/Au interfaces. (d) Equilibrium energy band diagram for MEH-PPV/CdSe sample. The vacuum levels are not shown. All the energies are with respect to the vacuum level. The doping natures of the semiconductors are unknown. 
dissociation of photogenerated excitons. The electrons and holes are spatially separated in the QDs and the polymer, respectively, by the built-in electric field arising from the energy band offsets of the QD and the polymer. There are different possible mechanisms of charge transport in the polymer/QD bulk heterojunctions. In one of the mechanisms, QDs supply the excitons and induce charge separation. QDs act as sensitizers or traps for the separated electrons, which undergo non-radiative recombination. ${ }^{26,27}$ The holes percolate into the polymer, and, thus, hole conduction is the main mechanism of charge transport. In another mechanism of charge transport through bulk heterojunctions, both electrons and holes are transported through physically separate channels, with electrons transporting through the QDs and the holes transporting through the polymer. Thus, the dominant mechanism of charge transport in these systems may depend on the concentration of QDs in the polymer. ${ }^{27}$ Regular drift and diffusion processes are also present. It is difficult to determine the probabilities of each of these transport processes.

In an effort to model the charge transport in a P3HT/ CdSe hybrid system, it was shown that the temperaturedependent hole mobility models are not adequate to fit the experimental data, but a good fit was obtained using trap models based on drift of holes under the influence of traps. ${ }^{28}$ The concentration of QDs in this system was low (50\% wt. of CdSe in P3HT). Thus, polymers form a percolating network for the holes, with hole conduction as a major conduction mechanism for low concentrations of QDs in the polymer. However, for larger concentrations of QDs in the polymer, nanocrystals provide additional pathways for electron transport ${ }^{27}$ by interdot tunneling and hoping mechanisms and could be a significant component of overall conduction. Based on tunneling mechanism, a simplest possible model to simulate the I-V characteristics in the two samples is a double barrier quantum well device (DBQWD), which consists of QDs sandwiched between polymer molecules. $^{29}$ This model is a good first approximation of the device. The real system is more complex. With the spin-casting technique followed to fabricate the two samples, it is less probable to have vertically aligned QDs in the polymer matrix to form a multi-quantum well structure. The transport model consists of a number of quantized energy levels depicting the QD island and tunneling barriers connected to macroscopic contacts from which electrons hop into available energy levels. The schematic of the DBQWD is shown in Fig. 7(a). The model is based on many body interactions and rate equations. ${ }^{29}$ It takes into account the electronelectron interactions and the electron-phonon interactions, which play important roles in small cross-sectional devices made of QDs. The detailed theoretical formulation of the model has been discussed by Klimeck. ${ }^{29}$ The quasibound energy levels in the MEH-PPV/CdSe DBQWD are computed to be at 0.16 and $0.60 \mathrm{eV}$ for a well width of $3 \mathrm{~nm}$ and depth of $0.9 \mathrm{eV}{ }^{29}$ The well depth is determined from the band offsets. The CdSe dots are approximately $3 \mathrm{~nm}$ in diameter. In Fig. 7(a) is displayed the I-V curve simulated using these two energy levels at $0.16 \mathrm{eV}$ and $0.60 \mathrm{eV}$, a chemical potential parameter $0.3 \mathrm{eV}$, a charging parameter 0.2 , the left contact coupling parameters 0.00003 and 0.00012 , and the right
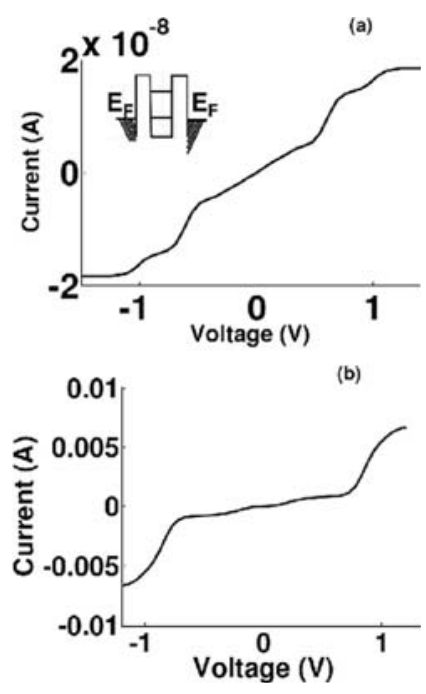

FIG. 7. I-V characteristics simulated using a NanoHub model on a single QD between two metal electrodes. (a) MEH-PPV/CdSe DBQWD energy levels $0.16 \mathrm{eV}$ and $0.60 \mathrm{eV}$, chemical potential $0.3 \mathrm{eV}$, charging parameter 0.2 , contact coupling parameters: left $0.00003,0.00012$ and right 0.00003 , 0.00012. (b) P3HT/CdSe DBQWD energy levels $0.13 \mathrm{eV}$ and $0.45 \mathrm{eV}$, chemical potential $0.65 \mathrm{eV}$, charging parameter 0.1 , contact coupling parameters: left 5, 50 and right 5, 50 .

contact coupling parameters 0.00003 and 0.00012 . The parameters are chosen to get a closest fit to the experimental curve. Comparison of Fig. 4(a) with Fig. 7(a) reveals that there is a close agreement between the magnitudes of currents. The quasibound energy levels in the P3HT/CdSe DBQWD computed for a well width of $3 \mathrm{~nm}$ and well depth of $0.5 \mathrm{eV}$ are at 0.13 and $0.45 \mathrm{eV}$. The simulated I-V curve for this system is displayed in Fig. 7(b). The parameters for this curve are: energy levels 0.13 and $0.45 \mathrm{eV}$; a chemical potential parameter $0.65 \mathrm{eV}$; a charging parameter 0.1 ; the left contact coupling parameters 5, 50; and the right contact coupling parameters 5,50 . The nature of this curve and magnitude of current is close to the experimental data shown in Fig. 5(a). Thus, electron tunneling based on a simple DBQWD model provides a good fit to the I-V curves in the two nanocomposite systems.

\section{CONCLUSIONS}

Charge transport has been investigated in MEH-PPV/ CdSe and P3HT/CdSe systems. Higher currents observed from the P3HT/CdSe as compared to a MEH-PPV/CdSe are analyzed with hole conduction through polymer and electron conduction through nanocrystals. Based on energy band offsets and ignoring the presence of additional band offsets, which may be present due to interfacial properties, a tunneling-based DBQWD model shows a good fit to experimental results.

${ }^{1}$ M. Zhu, T. Cui, and K. Varahramyan, Microelectron. Eng. 75, 269 (2004). ${ }^{2}$ S. C. Jain, M. Willander, and V. Kumar, Semicond. Semimetals 81, 1 (2007).

${ }^{3}$ A. L. Rogach and J. M. Lupton, "Hybrid OLEDs with semiconductor nanocrystals," in Organic Light Emitting Devices: Synthesis, Properties and Applications, edited by K. Mullen and U. Scherf (Wiley VCH, Berlin, 2006).

${ }^{4}$ A. L. Rogach, Semiconductor Nanocrystal Quantum Dots: Synthesis, Assembly, Spectroscopy and Applications (Springer, New York, 2008). 
${ }^{5}$ P. T. Snee, R. C. Somers, G. Nair, J. P. Zimmer, M. G. Bawendi, and D. G. Nocera, J. Am. Chem. Soc. 128(41), 13320 (2006).

${ }^{6}$ E. J. McCumiskey, N. Chandrasekhar, and C. R. Taylor, Nanotechnology 21, 225703 (2010).

${ }^{7}$ D. S. Ginger and N. C. Greenham, Phys. Rev. B 59, 10622 (1999).

${ }^{8}$ Y. Lin, C. Chen, J. Chang, T. Y. Lin, I. S. Liu, and W. Su, Nanotechnology 17, 1260 (2006).

${ }^{9}$ B. Carlson, K. Leschkies, E. S. Aydil, and X. Y. Zhu, J. Phys. Chem. C 112, 8419 (2008).

${ }^{10}$ X. Jiang, R. D. Schaller, S. B. Lee, J. M. Pietryga, V. I. Klimov, and A. A. Zakhidov, J. Mater. Res. 22(8), 2204 (2007).

${ }^{11}$ Y. Greenwald, X. Xu, M. Fourmigue, G. Srdanov, C. Koss, F. Wudl, and A. J. Heeger, J. Polym. Sci., Part A: Polym. Chem. 36, 3115 (1998).

${ }^{12}$ Y. Zhou, Y. Li, H. Zhong, J. Hou, Y. Ding, C. Yang, and Y. Li, Nanotechnology 17, 4041 (2006).

${ }^{13}$ I. H. Campbell, T. W. Hagler, D. L. Smith, and J. P. Ferraris, Phys. Rev. Lett. 76, 1990 (1996).

${ }^{14}$ J. Guo, H. Ohkita, H. Benten, and S. Ito, J. Am. Chem. Soc. 131, 16869 (2009).

${ }^{15}$ G. D. Scholes, Annu. Rev. Phys. Chem. 54, 57 (2003).

${ }^{16}$ F. Kong, Y. M. Sun, and R. K. Yuan, Nanotechnology 18, 265707 (2007).

${ }^{17}$ M. Pientka, V. Dyakonov, D. Meissner, A. Rogach, D. Talapin, H. Weller, L. Lutsen, and D. Vanderzande, Nanotechnology 15, 163 (2004).
${ }^{18}$ V. Shrotriya, J. Ouyang, R. J. Tseng, G. Li, and Y. Yang, Chem. Phys. Lett. 411, 138 (2005).

${ }^{19}$ D. M. DeLongchamp, B. M. Vogel, Y. Jung, M. C. Gurau, C. A. Richter, O. A. Kirillov, J. Obrzut, D. A. Fischer, S. Sambsivan, L. J. Richter, and E. K. Lin, Chem. Mater. 17, 5610 (2005).

${ }^{20}$ Q. Shi, Y. Hou, J. Lu, H. Jin, Y. Li, X. Sun, and J. Liu, Chem. Phys. Lett. 425, 353 (2006).

${ }^{21}$ Y. Lin, T. Zeng, W. Lai, C. Chen, Y. Lin, Y. Chang, and W. Su, Nanotechnology 17, 5781 (2006).

${ }^{22}$ V. Svrcek, I. Turkevych, and M. Kondo, Nanoscale Res. Lett. 4, 1389 (2009).

${ }^{23}$ N. S. Sariciftci, D. Braun, C. Zhang, V. I. Srdanov, A. J. Heeger, G. Stucky, and F. Wudl, Synth. Met. 59(3), 333 (1993).

${ }^{24}$ F. Xue, Z. Liu, Y. Su, and K. Varahramyan, Microelectron. Eng. 83, 298 (2006).

${ }^{25}$ K. Seki, T. Tani, and H. Ishii, Thin Solid Films 273(1/2), 20 (1996).

${ }^{26}$ Y. Wang and N. Herron, Phys. Lett. 200, 71 (1992).

${ }^{27}$ N. C. Greenham, X. Peng, and A. P. Alivisatos, Phys. Rev. B 54, 17628 (1996).

${ }^{28}$ K. Kumari, S. Chand, P. Kumar, S. N. Sharma, V. D. Vankar, and V. Kumar, Appl. Phys. Lett. 92, 263504 (2008).

${ }^{29} \mathrm{G}$. Klimeck, "Electron-Phonon and Electron-Electron Interactions in Quantum Transport," PhD Thesis (Purdue University, 1994), http:// nanohub.org/resources/3831. 